\title{
Electret Film-Enhanced Triboelectric Nanogenerator Matrix for Self- Powered Instantaneous Tactile Imaging
}

\author{
Ya Yang, ${ }^{\dagger} \ddagger$ Hulin Zhang, ${ }^{\ddagger}$ Xiandai Zhong, ${ }^{\dagger}$ Fang Yi, ${ }^{\ddagger}$, Ruomeng Yu, $^{\ddagger}$ Yue Zhang, ${ }^{\S}$ \\ and Zhong Lin Wang*, ${ }^{*},+$ \\ ${ }^{\dagger}$ Beijing Institute of Nanoenergy and Nanosystems, Chinese Academy of Sciences, Beijing 100083, China \\ ${ }^{\ddagger}$ School of Materials Science and Engineering, Georgia Institute of Technology, Atlanta, Georgia 30332-0245, United States \\ ${ }^{\S}$ School of Material Science and Engineering, University of Science and Technology Beijing, Beijing 100083, China
}

Supporting Information

ABSTRACT: We report the first self-powered electronic skin that consists of light-emitting diode (LED) and triboelectric nanogenerator (TENG) arrays that can be utilized for spatially mapping applied instantaneous-touch events and tracking the movement location of the target object by recording the electroluminescent signals of the LEDs without external power sources. The electret film-based TENG can deliver an open-circuit voltage of about $-1070 \mathrm{~V}$, a short-circuit current density of $10 \mathrm{~mA} / \mathrm{m}^{2}$, and a power density of $288 \mathrm{~mW} /$ $\mathrm{m}^{2}$ on an external load of $100 \mathrm{M} \Omega$. The LEDs can be turned on locally when the back surface of the active matrix is touched, and the intensity of the emitted light depends on the magnitude of the applied local pressure on the device. A

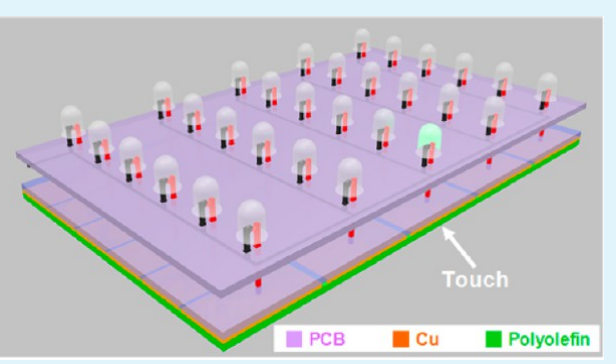
constructed active matrix of the LED-TENG array $(8 \times 7$ pixels $)$ can achieve self-powered, visual, and high-resolution tactile sensing by recording the electroluminescent signals from all of the pixels, where the active size of each pixel can be decreased to $10 \mathrm{~mm}^{2}$. This work is a significant step forward in self-powered tactile-mapping visualization technology, with a wide range of potential applications in touchpad technology, personal signatures, smart wallpapers, robotics, and safety-monitoring devices.

KEYWORDS: triboelectric nanogenerator, electronic skin, tactile sensor, self-powered systems, mechanical-energy harvesting

\section{INTRODUCTION}

Electronic skin has attracted increasing attention in the past decade for spatially mapping and quantifying various stimuli by using sensor arrays. ${ }^{1-3}$ In electronic skins, the electronic components such as thin-film transistors act as readout elements for controlling the current or voltage in each pixel. $^{4,5}$ The optimization of tactile sensors in electronic skins has been extensively investigated by utilizing some semiconductor/organic nanomaterials. ${ }^{6,7}$ Recently, a device density of 8464 pixels per $1 \mathrm{~cm}^{2}$ has been achieved using a two-terminal transistor-array matrix because of the piezotronic effect. ${ }^{8}$ Owing to the piezo-phototronic effect, the digital imaging of mechanical signals by optical means has been also demonstrated using piezoelectric $\mathrm{ZnO}$ nanowires to modulate the brightness of a light-emitting diode (LED) array. ${ }^{9}$ However, all of the reported electronic skins require an external power source to drive these tactile sensors. It is necessary to develop a self-powered electronic skin that is based on utilizing energy harvesters instead of external power sources or conventional batteries to drive the tactile sensors.

Currently, we have demonstrated an innovative approach to scavenge mechanical energy from impacts, sliding, and rotations by utilizing triboelectric nanogenerators (TENGs). ${ }^{10-14}$ Owing to the coupling between triboelectrification and electrostatic induction, the reported TENGs exhibit a good pressuresensitive characteristic. ${ }^{15}$ The output electric signal of the
TENGs can increase with an increase in the applied pressure; ${ }^{16}$ these TENGs have the dual functions of being both the pressure-sensitive media and the power sources in electronic skins, and they can be integrated into an active-matrix array. Each pixel in the matrix consists of a TENG with the electrode connected to one electrode of a LED, subsequently resulting in the LED turning on under an external applied pressure. By utilizing the LED-TENG array, the resulting system can provide tactile sensing, allowing the pressure profile to be both spatially mapped and visually observed.

In this article, we report a self-powered electronic skin that consists of the LED-TENG array that can be used to map applied instantaneous-touch events spatially and to track the movement location of the target object by a read out the electroluminescent signals of the LEDs without an external power source. The design principle makes use of the pressuresensitive TENG to modulate the local light-emitting intensity of the LED in each pixel. The map of the tactile distribution can be obtained by the analysis of the $2 \mathrm{D}$ light-emitting intensity distribution. The electret film-based TENG $\left(7 \times 7 \mathrm{~cm}^{2}\right)$ can produce an open-circuit voltage of about $-1070 \mathrm{~V}$ and a shortcircuit current density of $10 \mathrm{~mA} / \mathrm{m}^{2}$. When an instantaneous

Received: December 30, 2013

Accepted: February 14, 2014 

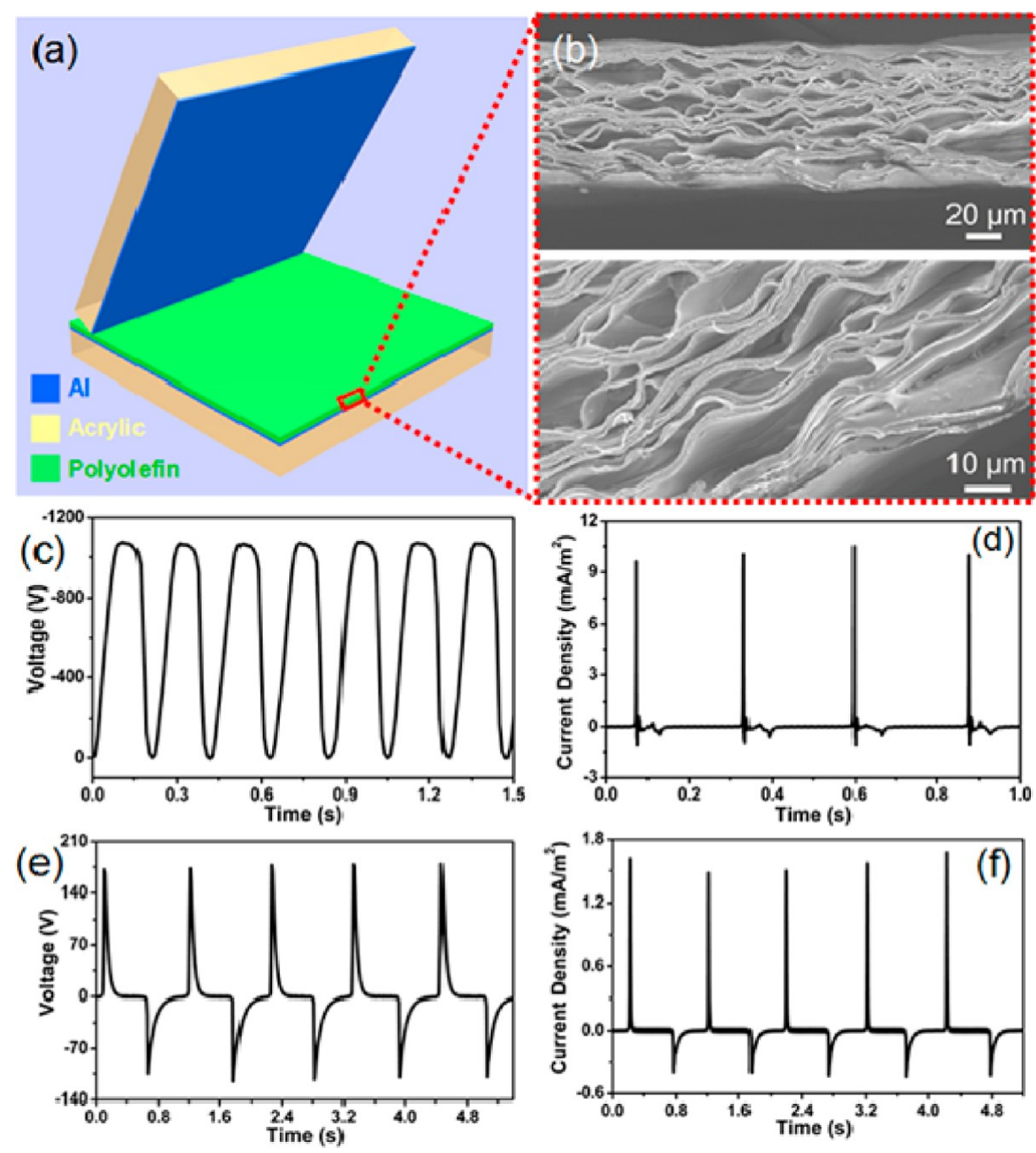

Figure 1. (a) Schematic diagram of the fabricated TENG. (b) Cross-sectional SEM image of the polyolefin film. (c, d) Measured open-circuit voltage (c) and short-circuit current density (d) of the TENG. (e, f) Measured output voltage (e) and output current density (f) of the TENG under a load resistance of $100 \mathrm{M} \Omega$.

touch is applied on the active matrix of LED-TENG array, the corresponding LEDs can be directly turned on, and the intensity of the emitted light quantifies the magnitude of the applied local pressure on the pixels. Moreover, an active matrix of the LED-TENG array $(8 \times 7$ pixels $)$ was designed to achieve self-powered, visual tactile sensing, where the active size of each pixel (the electrode size of each TENG unit) can be decreased to $10 \mathrm{~mm}^{2}$.

\section{RESULTS AND DISCUSSION}

As schematically illustrated in Figure 1a, the designed TENG is composed of an $\mathrm{Al}$ foil, an electret film, and the $\mathrm{Al}$ electrode deposited on the electret film. The Al foil attached on acrylic glass $\left(7 \times 7 \mathrm{~cm}^{2}\right)$ plays the dual roles of a triboelectric surface and an electrode. As displayed in Figure 1b, the electret film consists of air voids separated by thin polyolefin layers by stretching the film in two perpendicular directions. The total thickness of the film is about $90 \mu \mathrm{m}$, where each layer has a thickness of smaller than $100 \mathrm{~nm}$, as depicted in Figure 1b. The electret film was polarized via the corona method, ${ }^{17}$ which causes breakdowns in the voids and thus results in positive and negative charges on the two surfaces of the film, respectively. ${ }^{18,19}$ Figure 1c,d illustrates the output performance of the fabricated TENG, where the open-circuit voltage can reach $-1070 \mathrm{~V}$ with a peak current density of $10 \mathrm{~mA} / \mathrm{m}^{2}$.

Under a load resistance of $100 \mathrm{M} \Omega$, Figure 1e,f shows that the TENG can produce an output voltage of $180 \mathrm{~V}$ with a current density peak of $1.6 \mathrm{~mA} / \mathrm{m}^{2}$, resulting in an output power density of $288 \mathrm{~mW} / \mathrm{m}^{2}$. As illustrated in Figure S1, when the bottom surface of the electret film was reversed to the top surface of the film, the output voltage and the output current density of the TENG were significantly decreased to $30 \mathrm{~V}$ and $0.6 \mathrm{~mA} / \mathrm{m}^{2}$, respectively. Usually, the output performance of the TENG at the two surfaces resulting from the triboelectric effect should be similar because of the use of the same triboelectric materials. In this study, the large difference for the output voltage/current density of the TENGs at the two surfaces is associated with the different electrostatic charges in the electret film, where one surface has positive surface charges and the other surface has negative surface charges. The induced positive and negative charges at the two surfaces in the electret film can modulate the output performance of the TENG. One surface can enhance the output performance (Figure 1e,f), whereas the other one will reduce it (Figure S1). The results indicate that the output performance of the TENG can be effectively enhanced by utilizing a charged electret film. 
(a)

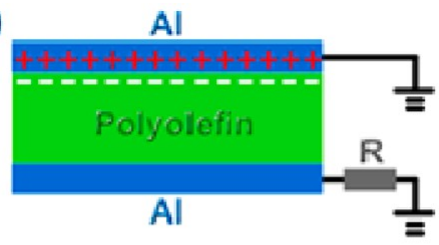

1
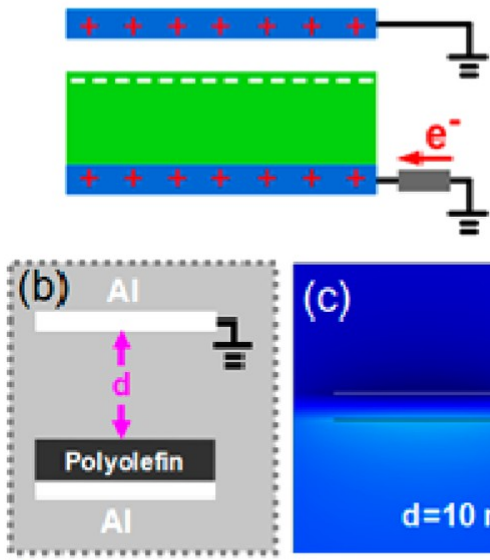

0

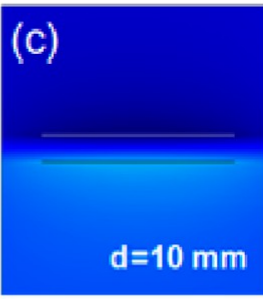

$-15$
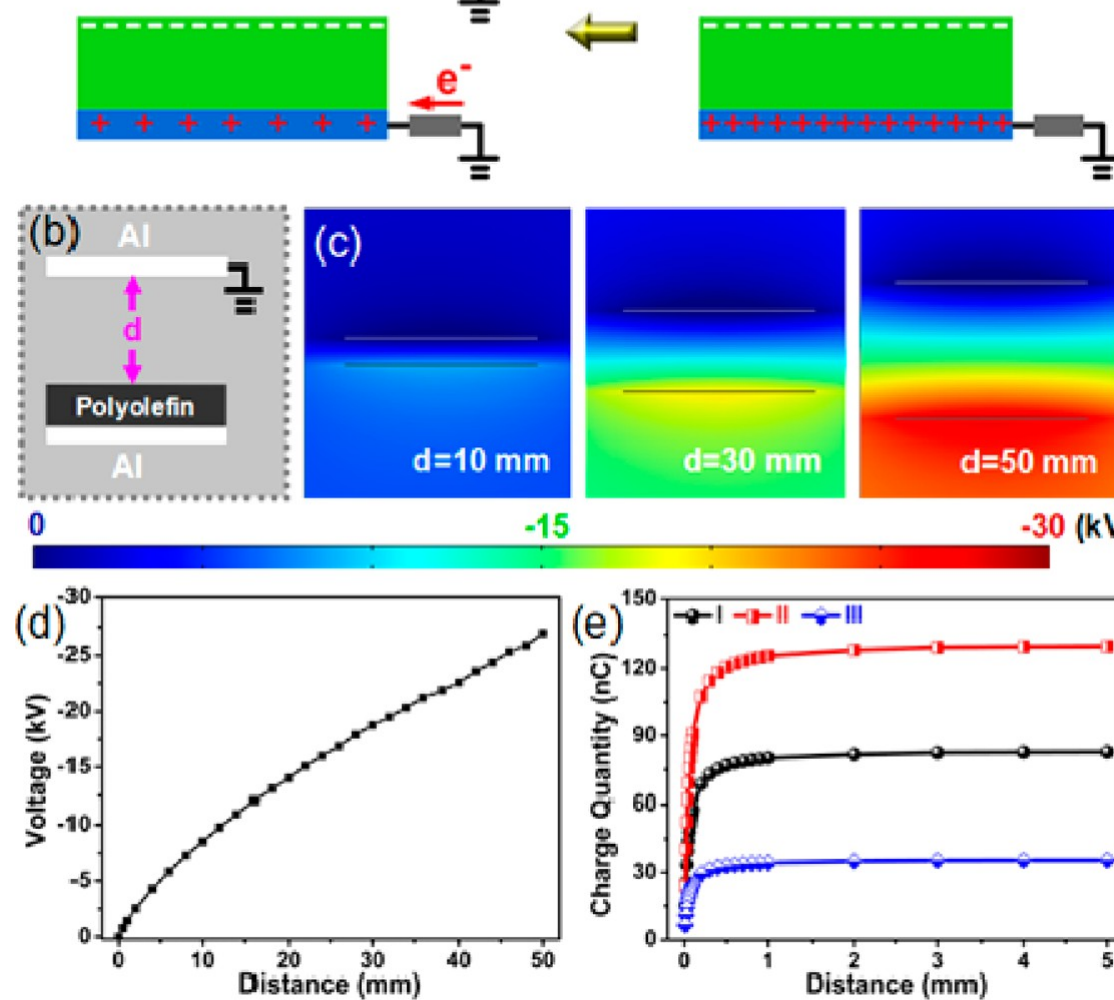

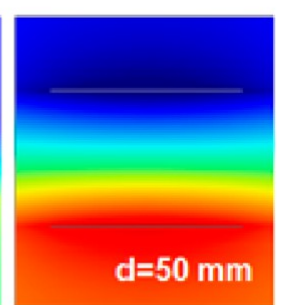

$-30(\mathrm{kV})$

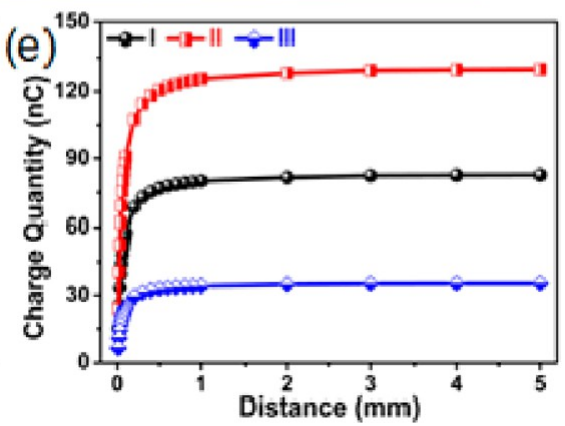

Figure 2. (a) Schematic diagram of the electricity-generation process in a full cycle because of the triboelectric effect. (b) Schematic diagram of the model for the calculation of the open-circuit conditions. (c) Finite-element simulation of the potential distribution in the TENG under different separation distances. (d) Calculated potential of the bottom Al electrode under different separation distances. (e) Calculated total charges on the bottom $\mathrm{Al}$ electrode under different separation distances: I, triboelectric charges; II, triboelectric charges and electrostatic charges (film top, -; film bottom, +; triboelectric charge density, $-17.5 \mu \mathrm{C} / \mathrm{m}^{2}$; electrostatic charge density, $\pm 10 \mu \mathrm{C} / \mathrm{m}^{2}$ ); and III, triboelectric charges and electrostatic charges (film top, +; film bottom, -).

Detailed calculations using COMSOL can be utilized to describe how the different surface charges can affect the output performance of the device. The difference in the shapes of the curves between Figure 1, panels $c$ and $d$ and panels e and $f$ is associated with the different loading resistances.

Figure 2a illustrates the working principle of the fabricated TENG by the coupling of contact electrification and electrostatic induction because of the triboelectric effect. In the original position, the surfaces of the $\mathrm{Al}$ foil and the polyolefin film fully contact each other, resulting in electrons injected from the $\mathrm{Al}$ to the polyolefin film because of the more triboelectrically negative characteristic of the polyolefin film. There is no electron flow in the external circuit because the produced triboelectric charges with opposite polarities are fully screened, which is the contact electrification process. Once there is a relative separation between the $\mathrm{Al}$ foil and the polyolefin film, these triboelectric charges can not be compensated. The negative triboelectric charges on the polyolefin film can induce positive charges on the bottom $\mathrm{Al}$ electrode, driving free electrons to flow from the bottom $\mathrm{Al}$ electrode to the ground. By increasing the separation distance between the $\mathrm{Al}$ foil and the polyolefin film, the negative triboelectric charges on the polyolefin film can be fully screened from the induced positive charges on the bottom $\mathrm{Al}$ electrode, resulting in no output signals that can be observed. Furthermore, when the $\mathrm{Al}$ foil is reverted to approach the polyolefin film, the induced positive charges on the bottom $\mathrm{Al}$ electrode decrease, driving the electrons to flow from the ground to the bottom $\mathrm{Al}$ electrode, which is the electrostatic induction process. There is no observed output voltage/current until the $\mathrm{Al}$ foil and the polyolefin film fully contact each other 

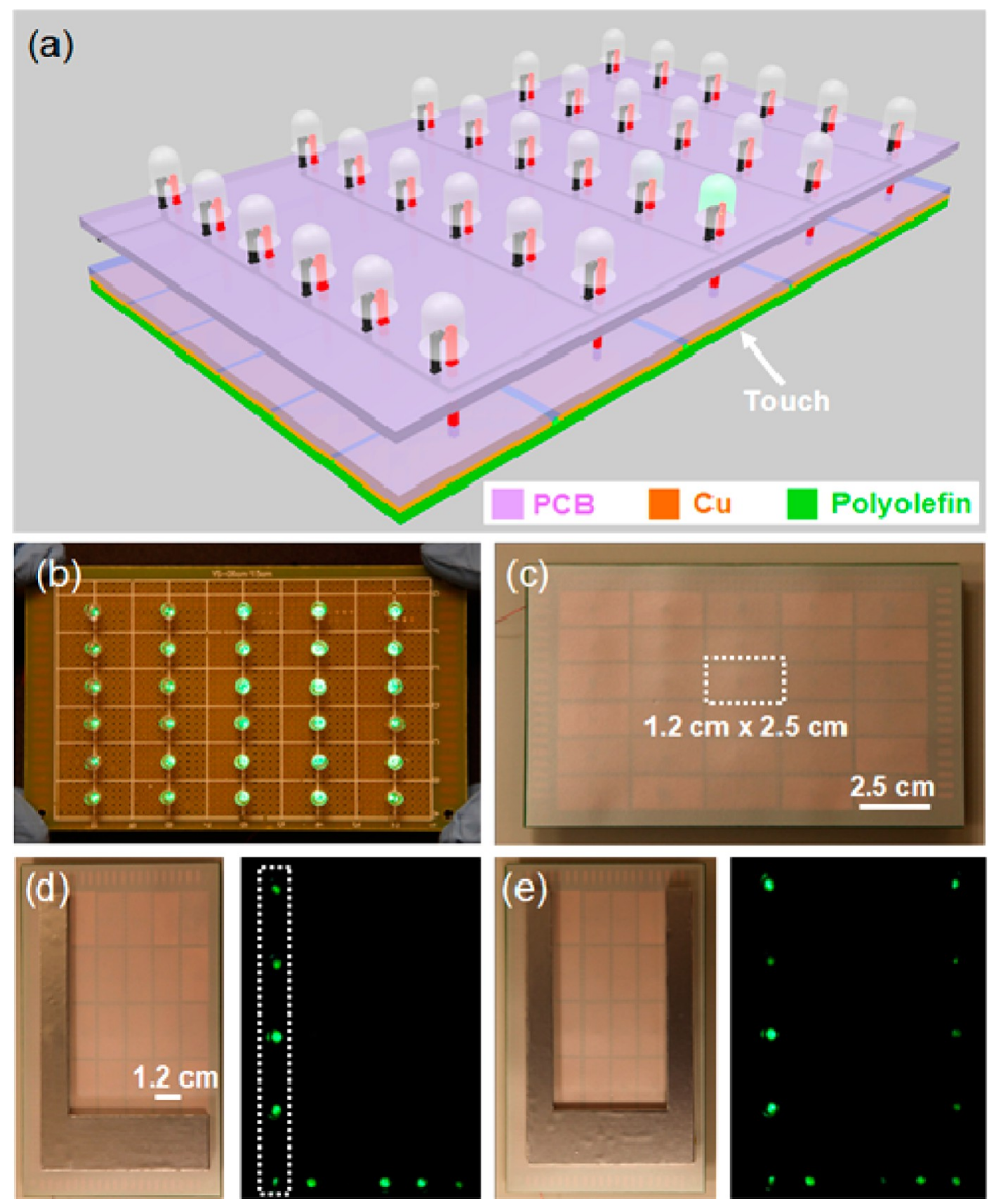

Figure 3. (a) Schematic diagram of the self-powered electronic skin capable of spatially mapping and visually displaying the applied-touch events. The inset shows a photograph of the fabricated device. (b) Photograph of all of the lighted LEDs in the electronic skin, indicating that all of the LEDs are functional. (c) Photograph of the TENG array in the electronic skin showing that each pixel has a size of $1.2 \times 2.5 \mathrm{~cm}$. $(\mathrm{d}$, e) Electroluminescence images of the device when pressure with (d) L- and (e) U-shaped Al slabs was applied to it, where the optical images were recorded under the touching frequency of about $0.5 \mathrm{~Hz}$.

again. This is a full cycle of the triboelectric effect-induced electricity-generation process of the TENG.

The electric potential distribution in the TENG can be verified through numerical simulation using COMSOL. The proposed model is based on an $\mathrm{Al}$ foil, a polyolefin film, and a bottom $\mathrm{Al}$ electrode with dimensions of $7 \mathrm{~cm} \times 7 \mathrm{~cm}$ and a thickness of $90 \mu \mathrm{m}$ for the polyolefin film and $50 \mu \mathrm{m}$ for the $\mathrm{Al}$ foil and the bottom $\mathrm{Al}$ electrode, as displayed in Figure 2b. The triboelectric charge density on the polyolefin film was assumed to be $-17.5 \mu \mathrm{C} / \mathrm{m}^{2}$, and the $\mathrm{Al}$ foil was connected with the ground. As depicted in Figure $2 c, d$, the calculated results of the electric potential distribution in the TENG under the different separation distances between the polyolefin film and the $\mathrm{Al}$ foil clearly show that the electric potential on the bottom $\mathrm{Al}$ electrode for the open-circuit condition increases with the increasing separation distance. To obtain a more quantitative understanding about charge transfer in the TENG, the bottom $\mathrm{Al}$ electrode was connected with the ground, as illustrated in Figure S2. The curve I in Figure 2e shows that the amount of the total charges on the bottom $\mathrm{Al}$ electrode increases with the increasing separation distance between the $\mathrm{Al}$ foil and the polyolefin film and approaches a saturation value when the distance is larger than $5 \mathrm{~mm}$. To avoid the effect of separation distance on the performance of the device in Figure 2e, all of 

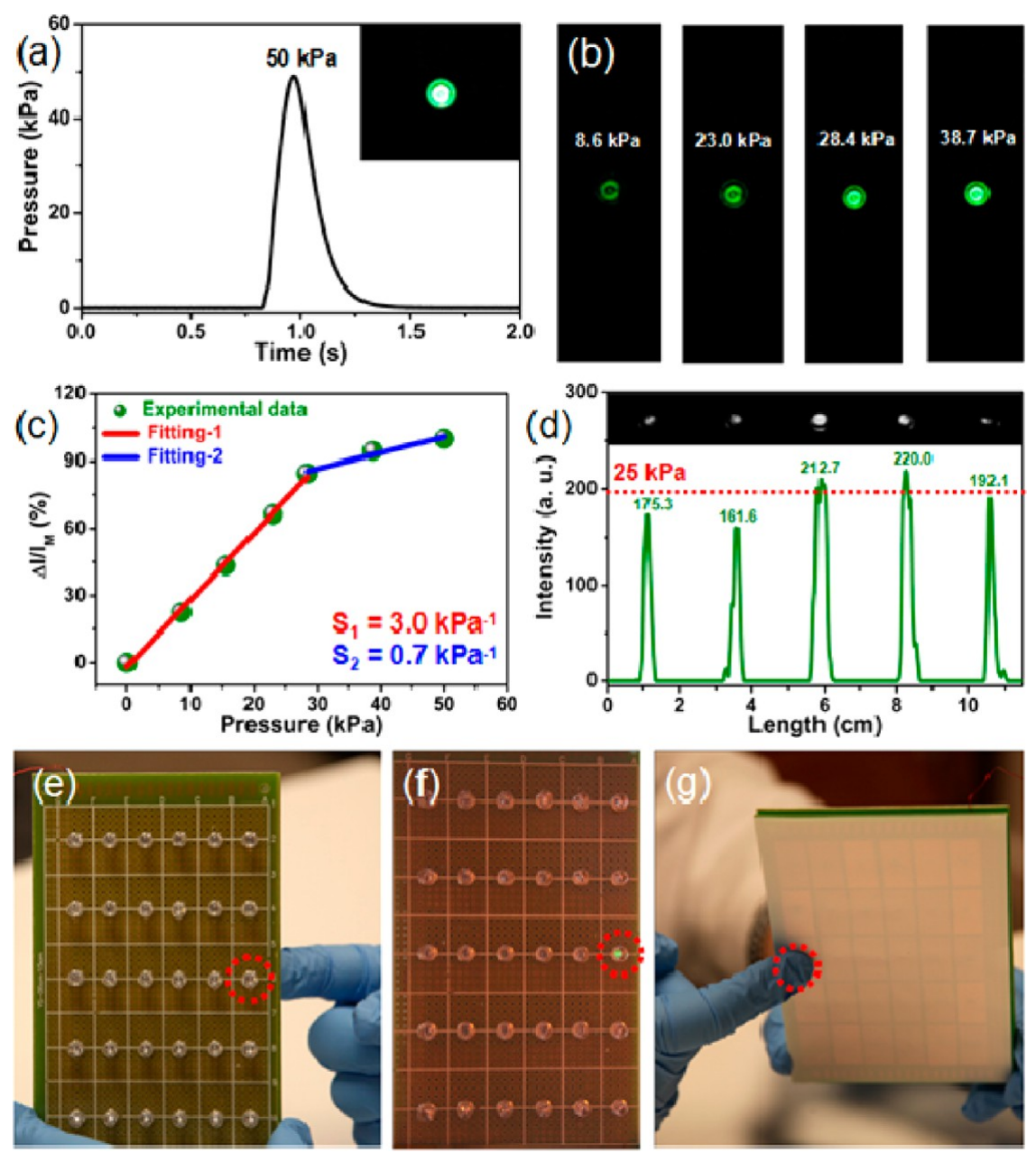

Figure 4. (a) Measured electroluminescence spectra of a single-lighted LED. The inset illustrates a photograph of the LED. (b) Optical images of the light-emission peaks of the LED under different pressure pulses. (c) Modulation factor $\left(M=(\Delta I) / I_{M}\right)$ of a LED-TENG pixel as a function of the applied instantaneous pressure. (d) Light-emission densities of the five pixels in the area marked with white color in Figure $3 \mathrm{c}$, indicating an average instantaneous pressure of about $25 \mathrm{kPa}$. (e-g) Optical images of the electronic skin that was touched using a human finger covered with a nitrile glove.

the measurements were achieved under a separation distance larger than $10 \mathrm{~mm}$. To understand the effect of the electrostatic charges in the electret film on the charge transfer in the TENG, Figures S3 and S4 show the electron-transfer principles for the two kinds of electrostatic charge distributions in the electret film, respectively. Once there is a separation distance between the $\mathrm{Al}$ foil and the electret film, the electrons can flow along opposite directions for the two kinds of electrostatic charge distributions, respectively. When the flow direction of the electrons for the electrostatic device is same as that of the TENG, there are more electrons that can be transferred between the $\mathrm{Al}$ electrode and the ground, as displayed in the curve II in Figure 2e. If the current direction of the electrostatic device is opposite as compared with that of the TENG, then the amount of transferred electrons can be decreased, as shown in the curve III in Figure 2e. The results indicate that the electrostatic charges in the electret film can be utilized to enhance the output performance of the TENG.

To realize the finger/hand touch-sensing functionality of the device, we also measured output performance of another TENG, where the human hand covered with a nitrile glove was used to replace the top $\mathrm{Al}$ foil in Figure $2 \mathrm{a}$, as shown in Figure S5. The purpose of utilizing the nitrile glove is to avoid the instability of triboelectric characteristics of human hands resulting from perspiration or other cases. The working principle and the calculated electron-transfer process are shown in Figure S6 and indicate that the electrons can flow from the bottom $\mathrm{Al}$ electrode to ground when the separation distance between the nitrile glove and the polyolefin film increases, whereas the electrons can flow along the opposite direction for the decrease in the separation distance. To demonstrate that the fabricated TENG can be used as a power source to light up commercial LEDs, 40 green LEDs were divided into two groups in series with the opposite polarities, which were both connected to the TENG. When the device was compressed by the $\mathrm{Al}$ foil or the hand covered with a nitrile glove, one group of LEDs can be simultaneously lit up because of the produced positive voltage/current peak, as displayed in Movie 1 (Supporting Information). When the compressive force was released, the other group of the LEDs can be lit up because of the produced negative voltage/current peak. With an increase in the touch frequency, all of the LEDs can be simultaneously lit up because of the high switching frequency, as illustrated in Figure S7a. To understand the size effect of the TENG on the brightness of the connected LED, we fabricated different TENGs with sizes of $2 \times 2,3 \times 3,4 \times 4,5 \times 5$, and 6 


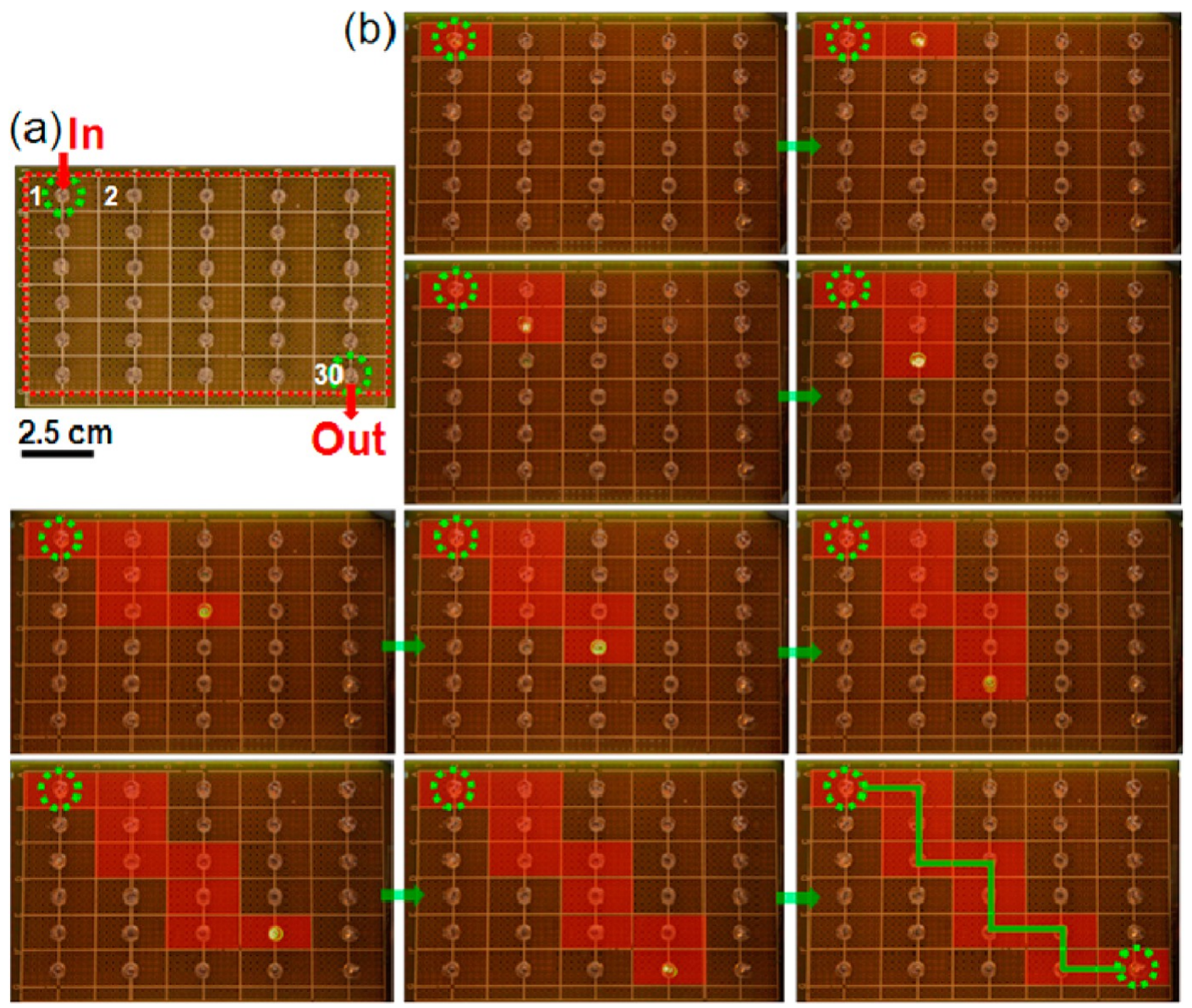

Figure 5. (a) Photograph of the device for tracking the motion route of the target object, where pixels 1 and 30 are regarded as the entrance and exit ports, respectively. (b) Obtained mapping images of the electroluminescence signals from all of the pixels indicate that an object moves along $1 \rightarrow 2$ $\rightarrow 7 \rightarrow 12 \rightarrow 13 \rightarrow 18 \rightarrow 23 \rightarrow 24 \rightarrow 29 \rightarrow 30$

$\times 6 \mathrm{~cm}^{2}$, where each TENG was connected with one LED, as depicted in Figure S7b,c. Figure S7d shows the photograph of these LEDs lit by TENGs under the same pressure, indicating that all the LEDs can be lit up and the brightness of the LEDs decreases with the decreasing size of TENG, as illustrated in Figure S7e. These results suggest the possibility of device construction for a self-powered tactile-sensor system that utilizes the TENG pixels to drive LEDs to realize the applied instantaneous pressure visualization.

Figure 3a illustrates a schematic diagram of the fabricated self-powered electronic skin. First, an LED array was fabricated on a printed circuit board (PCB) substrate, where all of the cathode electrodes of the LEDs were connected to the ground. Next, each anode electrode of the LED was connected to one $\mathrm{Cu}$ electrode of the TENG at the bottom. A layer of the polyolefin film was then covered on the $\mathrm{Cu}$ electrode array. Although the size of each pixel is limited to that of the $\mathrm{Cu}$ electrode, this fabrication approach can result in highly uniform and large-scale production of the active-sensor array by utilizing well-established microfabrication process technology. As displayed in Figure $3 \mathrm{~b}$, the photograph of the fabricated device illustrates that the active matrix of the LED-TENG array has 6 $\times 5$ pixels and all of the LEDs are functional. Figure $S 8$ depicts the electroluminescence spectra of the LED with the major emission peak located at $513 \mathrm{~nm}$, where the photograph of the lighted LED is shown in the inset. As shown in Figure 3c, the optical image of the back side of the device clearly displays that each pixel has a size of $1.2 \mathrm{~cm} \times 2.5 \mathrm{~cm}$. The fabricated device can be utilized to map spatially and to display visually the applied-touch profile. As displayed in Figure 3d, applying pressure through the L-shaped $\mathrm{Al}$ slab at the bottom of the electronic skin can produce recognizable letter $\mathrm{L}$ on the surface of the device, which can be also seen in Movie 2 (Supporting Information). Figures $3 \mathrm{e}$ and $\mathrm{S} 9$ illustrate that the fabricated electronic skin can be also utilized to map spatially and to display visually the touch profiles applied by the U- and Eshaped Al slabs, respectively.

When an instantaneous pressure is applied on the back side of the active matrix of the LED-TENG array, the pressure peak can be determined by utilizing a commercial pressure sensor, and the corresponding light-emission-intensity peaks of the LEDs can be confirmed using a camera-imaging technique. To avoid the effect of the loading rate of the pressure on the output performance of TENG and the brightness of the LED, the pressure measurements were achieved under the almost same touching frequency of about $0.5 \mathrm{~Hz}$. As illustrated in Figure 4a, when the applied pressure peak is about $50 \mathrm{kPa}$, the obtained light-emission peak is displayed in the inset. It was found that the obtained light-emission-intensity peak decreases with decreasing the applied instantaneous pressure, as depicted in Figure 4b. The light-emission intensity of each LED-TENG pixel can be largely modulated by the magnitude of the applied pressure. The brightness of the background signal without the applied pressure on the device is denoted $I_{0}$. The emission 

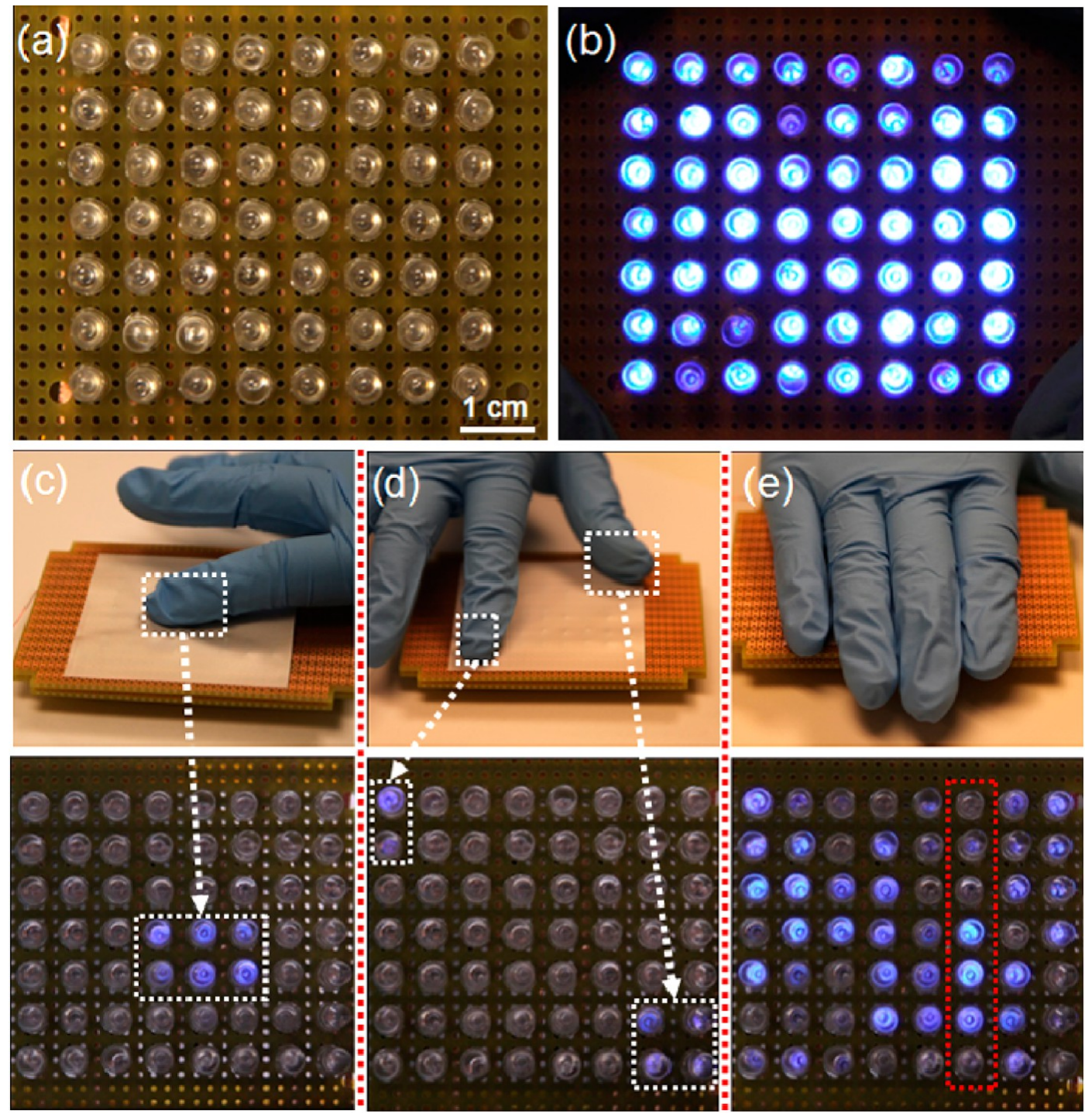

Figure 6. (a) Photograph of the fabricated active matrix of the LED-TENG array $(8 \times 7$ pixels). (b) Photograph of all of the lighted blue LEDs in the electronic skin, indicating that all of the blue LEDs are functional. $(c-e)$ Obtained mapping figures of the electroluminescence signals from all of the pixels when (c) one finger, (d) two fingers, and (e) one hand covered with a nitrile glove were utilized to touch the device from the back side, respectively. (f) Light-emission densities of the seven pixels in the area marked with red color in panel e, indicating that the largest pressure appears in the fourth pixel.

intensity of the LED under the applied pressure is assigned to $I_{\mathrm{p}}$, and the largest brightness value is $I_{M}$. All of the obtained images were converted to gray images for the analysis of the light-emission intensities. The modulation factor, $M$, of LED intensity is then defined as $M=\left(I_{P}-I_{0}\right) / I_{M}$. The sensitivity, $S$, of the applied pressure can be expressed as

$$
S=\left(\Delta I / I_{M}\right) / \sigma=M / \sigma
$$

where $M$ is the modulation factor and $\sigma$ is the applied pressure. The measured instantaneous pressure and emission intensity peak data of the LED are displayed in Figures S10 and S11, respectively. From the slopes of the linear responses in Figure $4 \mathrm{c}$, the sensitivities of pressure can be extracted to be about 3.0 $\pm 0.1 \mathrm{kPa}^{-1}$ from 0 to $30 \mathrm{kPa}$ and $0.7 \pm 0.1 \mathrm{kPa}^{-1}$ from 30 to $50 \mathrm{kPa}$, which are much larger than that of the reported value. 9 By the analysis of the obtained light-emission intensities of the LEDs at five pixels in Figure 3d, the applied average pressure on the device can be extracted to be about $25 \mathrm{kPa}$, which is consistent with the measured average pressure data of about 28
$\mathrm{kPa}$ by using a commercial pressure sensor, as illustrated in Figure S12.

Figure $4 \mathrm{e}-\mathrm{g}$ shows the optical images of the electronic skin under the touching of a finger tip covered with a nitrile glove, indicating that finger touch can be utilized to light up the LED directly. As displayed in Figure S13a, when an instantaneous pressure was applied on the center area of the sensor system using a human hand covered with a nitrile glove, the corresponding LEDs can be lit up, as illustrated in Figure $\mathrm{S} 13 \mathrm{~b}$. It was found that the largest pressure was applied on the fourth LED-TENG pixel by the analysis of the emission densities of all of the LEDs, as displayed in Figure S13c,d. The self-powered electronic skin can be utilized for sensing the touch-induced instantaneous pressure of the human finger/ hand covered with a nitrile glove, which can be also seen in Movie 3 (Supporting Information).

By addressing and monitoring the electroluminescent signals of the LEDs for all of the pixels in the active matrix, the electronic skin can be also utilized to detect the motion route of 
an object by recording light-emission mapping signals. As illustrated in Figure 5a, to demonstrate the use of the electronic skin for tracking the motion route of a target object, the positions at pixels 1 and 30 are regarded as the entrance and exit ports, respectively. When the LED on pixel 1 was lit up, we began to record the light-emission mapping figures of all of the pixels. The corresponding mapping figure recording was finished when the LED on pixel 30 was lit up. By analysis of the obtained mapping figures, we can determine how the object moved in the active matrix of the LED-TENG array $(6 \times 5$ pixels). As depicted in Figure 5b, the motion route of the object was determined to be $1 \rightarrow 2 \rightarrow 7 \rightarrow 12 \rightarrow 13 \rightarrow 18 \rightarrow 23 \rightarrow$ $24 \rightarrow 29 \rightarrow 30$ by analysis of the recorded 10 mapping figures. The electronic skin technology not only provides the selfpowered visualization functionality of the touch sensing and motion route tracking but also realizes a concealment monitoring characteristic because the light emission of the LEDs and the target object appear at the two sides of the sensor system, respectively.

As shown in Figure 6a, to enhance the spatial resolution of self-powered electronic skin, we fabricated another activematrix LED-TENG array with $8 \times 7$ pixels, where the active size (the electrode area of TENG) of each pixel can be decreased to $10 \mathrm{~mm}^{2}$, as displayed in Figure S14. As depicted in Figure $6 \mathrm{~b}$, the electroluminescence spectra of the LED exhibits a major emission peak at $456 \mathrm{~nm}$, where all of the blue LEDs are functional under an external bias, as shown in the inset. Figure $6 c$ displays that six blue LEDs can be lit up when a finger tip covered with a nitrile glove was used to touch the back surface of the active matrix. When the instantaneous touches were simultaneously applied along the diagonal line of the matrix by using two fingers, the responding LEDs were lit up at two positions, as illustrated in Figure 6d. As shown in Figure 6e, when all 56 pixels were touched by utilizing the human hand covered with a glove, the obtained mapping figure clearly depicts that all of the LED-TENG pixel units are functional and the largest pressure appears at the fourth pixel in the area marked with red color by the analysis of the light-emission density distribution of the seven LEDs, as displayed in Figure S15. As depicted in Movie 4 (Supporting Information), we have demonstrated the methodology for fabricating a self-powered electronic skin for sensing touch-induced instantaneous pressure and visualization. Miniaturization of pixel size for a higher spatial resolution is also possible by using other techniques to produce a micro/nanoscale LED-TENG array, where the mutilayer electret films and micro/nanoscale LEDs can be utilized to increase the pixel resolution further. The potential challenges include how to fabricate the micro/ nanoscale LEDs and how to use the micro/nanoscle TENG to drive the corresponding LEDs. Other electret-film materials can be also used for fabricating the TENGs. The output performance of the TENG can be also enhanced by decreasing the thickness of the electret film. As compared with reported electronic skins, the advantages of this technology include low cost, simple structure, tactile visualization, and self-powered capability.

\section{CONCLUSIONS}

We have demonstrated a self-powered electronic skin consisting of a LED-TENG array that can be utilized for spatially mapping the applied instantaneous tactile events and tracking the movement location of the target object by recording the electroluminescent signals of the LEDs without external power sources. The electrostatic charges in the electret film can be utilized to enhance the output performance of the TENG. The LEDs can be lit up locally when an instantaneous pressure is applied on the active matrix, where the light-emission density of the LED quantifies the magnitude of the applied pressure. An active matrix of the LED-TENG array with the $8 \times 7$ pixels was utilized to demonstrate the self-powered, visual, and highresolution capabilities of the tactile/touch sensing by recording the electroluminescent signals from all of the pixels, where the active size of each pixel can be decreased to $10 \mathrm{~mm}^{2}$. Selfpowered electronic skin technology has a wide range of potential applications in touchpad technology, smart wallpaper, robotics, and safety-monitoring devices.

\section{EXPERIMENTAL SECTION}

Fabrication of the Self-Powered Sensor System. The fabricated TENG consists of an $\mathrm{Al}$ foil with a thickness of $50 \mu \mathrm{m}$, an electret film with a thickness of $90 \mu \mathrm{m}$, and an $\mathrm{Al}$ electrode deposited on the electret film. The electret film consists of air voids separated by thin polyolefin layers and was polarized via the corona method, resulting in positive and negative charges on the two surfaces of the film. The self-powered sensor system is composed of LED array layer, a TENG electrode layer, and the electret film. The LED array was fabricated on a PCB substrate, where all of the cathode electrodes of the LEDs were connected to the ground. Each anode electrode of the LED was connected with a $\mathrm{Cu}$ electrode, which is also the electrode of the TENG. The polyolefin film was then covered on the $\mathrm{Cu}$ electrode array. The size of each pixel was determined by the size of the TENG electrode, where the spatial resolution of the sensor system can be enhanced by decreasing the size of the TENG electrode. Pressure was applied on the bottom of the polyolefin film, and the LED can be turned on locally at the same time.

Measurement of the Self-Powered Sensor System. Under a load resistance of $100 \mathrm{M} \Omega$, a Keithley 6514 system electrometer and a low-noise current preamplifier (Stanford Research SR570) were used to measure the output voltage and the output current of TENG, respectively. The electroluminescent signals of the LEDs were recorded using a fiber-optic spectrometer. The light-emission intensities of the LEDs under the applied external pressures were monitored by using movie image acquisition and processing technologies. For pressure calibration, the applied instantaneous pressure was measured using a commercial pressure senor.

\section{ASSOCIATED CONTENT}

\section{S Supporting Information}

Output voltage and output current of the TENG when the polyolefin film was reversed for the two surfaces; constructed model for the calculation of the charge quantity on the bottom $\mathrm{Al}$ electrode under the different separation distances between the $\mathrm{Al}$ foil and the polyolefin film; working principle of the electrostatic device; output voltage and current of the TENG when the human hand covered with a nitrile glove and the polyolefin film were used for contact and separation; working principle of the TENG and the corresponding calculation for the charge quantity on the bottom $\mathrm{Al}$ electrode under the different separation distances between the human hand covered with a nitrile glove and the polyolefin film; photograph of the TENG lighting up 40 green LEDs; size effect of the TENG on the brightness of the LED; measured electroluminescence spectra of a single-lighted LED; electroluminescence image of the device when touched with an E-shaped Al slab; different pressure pulses measured from Figure $4 \mathrm{~b}$; light-emission density peak of the LED under the different pressure pulses; measured pressure applied on the LED-TENG array; lightemission distribution of the LEDs when a human hand covered 
with a nitrile glove was utilized to apply pressure on the LEDTENG array; photograph of the electrode array of the TENGs in the sensor system; and light-emission densities of the seven pixels in the area marked with red color in Figure 6e. This material is available free of charge via the Internet at http:// pubs.acs.org.

\section{AUTHOR INFORMATION}

\section{Corresponding Author}

*E-mail: zlwang@gatech.edu.

\section{Notes}

The authors declare no competing financial interest.

\section{ACKNOWLEDGMENTS}

This research was supported by the U.S. Department of Energy, Office of Basic Energy Sciences (DE-FG02-07ER46394), the Knowledge Innovation Program of the Chinese Academy of Sciences (KJCX2-YW-M13), and the Thousands Talents Program for a Pioneer Researcher and His Innovation Team, China.

\section{REFERENCES}

(1) Cao, Q.; Kim, H.; Pimparkar, N.; Kulkarni, J. P.; Wang, C.; Shim, M.; Roy, K.; Alam, M. A.; Rogers, J. A. Medium-Scale Carbon Nanotube Thin-Film Integrated Circuits on Flexible Plastic Substrates. Nature 2008, 454, 495-500.

(2) Sekitani, T.; Yokota, T.; Zschieschang, U.; Klauk, H.; Bauer, S.; Takeuchi, K.; Takamiya, M.; Sakurai, T.; Someya, T. Organic Nonvolatile Memory Transistors for Flexible Sensor Arrays. Science 2009, 326, 1516-1519.

(3) Someya, T.; Sekitani, T.; Iba, S.; Kato, Y.; Kawaguchi, H.; Sakurai, T. A Large-Area, Flexible Pressure Sensor Matrix with Organic FieldEffect Transistors for Artificial Skin Applications. Proc. Natl. Acad. Sci. U.S.A. 2004, 101, 9966-9970.

(4) Takei, K.; Takahashi, T.; Ho, J. C.; Ko, H.; Gillies, A. G.; Leu, P. W.; Fearing, R. S.; Javey, A. Nanowire Active-Matrix Circuitry for LowVoltage Macroscale Artificial Skin. Nat. Mater. 2010, 9, 821-826.

(5) Vishniakou, S.; Lewis, B. W.; Niu, X.; Kargar, A.; Sun, K.; Kalajian, M.; Park, N.; Yang, M.; Jing, Y.; Brochu, P.; Sun, Z.; Li, C.; Nguyen, T.; Pei, Q.; Wang, D. Tactile Feedback Display with Spatial and Temporal Resolutions. Sci. Rep. 2013, 3, 2521-1-2521-7.

(6) Wang, C.; Hwang, D.; Yu, Z.; Takei, K.; Park, J.; Chen, T.; Ma, B.; Javey, A. User-Interactive Electronic Skin for Instantaneous Pressure Visualization. Nat. Mater. 2013, 12, 899-904.

(7) Lipomi, D. J.; Vosgueritchian, M.; Tee, B. C.-K.; Hellstrom, S. L. Skin-Like Pressure and Strain Sensors Based on Transparent Elastic Films of Carbon Nanotubes. Nat. Nanotechnol. 2011, 6, 788-792.

(8) Wu, W.; Wen, X.; Wang, Z. L. Taxel-Addressable Matrix of Vertical-Nanowire Piezotronic Transistors for Active and Adaptive Tactile Imaging. Science 2013, 340, 952-957.

(9) Pan, C.; Dong, L.; Zhu, G.; Niu, S.; Yu, R.; Yang, Q.; Liu, Y.; Wang, Z. L. High-Resolution Electroluminescent Imaging of Pressure Distribution Using a Piezoelectric Nanowire LED Array. Nat. Photonics 2013, 7, 752-758.

(10) Fan, F.-R.; Tian, Z.-Q.; Wang, Z. L. Flexible Triboelectric Generator. Nano Energy 2012, 1, 328-334.

(11) Zhang, X.-S.; Han, M.-D.; Wang, R.-X.; Zhu, F.-Y.; Li, Z.-H.; Wang, W.; Zhang, H.-X. Frequency-Multiplication High-Output Triboelectric Nanogenerator for Sustainably Powering Biomedical Microsystems. Nano Lett. 2013, 13, 1168-1172.

(12) Li, W.; Sun, J.; Chen, M. Triboelectric Nanogenerator Using Nano-Ag Ink as Electrode Material. Nano Energy 2013, 3, 95-101.

(13) Yang, Y.; Zhang, H.; Chen, J.; Jing, Q.; Zhou, Y. S.; Wen, X.; Wang, Z. L. Single-Electrode-Based Sliding Triboelectric Nanogenerator for Self-Powered Displacement Vector Sensor System. ACS Nano 2013, 7, 7342-7351.
(14) Zhang, H.; Yang, Y.; Zhong, X.; Su, Y.; Zhou, Y.; Hu, C.; Wang, Z. L. Single-Electrode-Based Rotating Triboelectric Nanogenerator for Harvesting Energy from Tires. ACS Nano 2013, 8, 680-689.

(15) Lin, L.; Xie, Y.; Wang, S.; Wu, W.; Niu, S.; Wen, X.; Wang, Z. L. Triboelectric Active Sensor Array for Self-Powered Static and Dynamic Pressure Detection and Tactile Imaging. ACS Nano 2013, 7, 82668274.

(16) Bai, P.; Zhu, G.; Lin, Z.-H.; Jing, Q.; Chen, J.; Zhang, G.; Ma, J.; Wang, Z. L. Integrated Multilayered Triboelectric Nanogenerator for Harvesting Biomechanical Energy from Human Motions. ACS Nano 2013, 7, 3713-3719.

(17) Zhong, Q.; Zhong, J.; Hu, B.; Hu, Q.; Zhou, J.; Wang, Z. L. A Paper-Based Nanogenerator as a Power Source and Active Sensor. Energy Environ. Sci. 2013, 6, 1779-1784.

(18) Zhang, X.; Hillenbrand, J.; Sessler, G. M. Ferroelectrets with Improved Thermal Stability Made from Fused Fluorocarbon Layers. J. Appl. Phys. 2007, 101, 054114.

(19) Sessler, G. M.; Hillenbrand, J. Electromechanical Response of Cellular Electret Films. Appl. Phys. Lett. 1999, 75, 3405-3407. 